\title{
Long-Term Levels of LDL-C and Cognitive Function: The CARDIA Study
}

\author{
Matthew T. Mefford ${ }^{1}$ (i) , Ligong Chen ${ }^{1}$, Cora E. Lewis ${ }^{1}$, Paul Muntner ${ }^{1}$, Stephen Sidney ${ }^{2}$, Lenore J. Launer ${ }^{3}$, Keri L. Monda ${ }^{4}$, \\ Andrea Ruzza ${ }^{5}$, Helina Kassahun ${ }^{5}$, Robert S. Rosenson ${ }^{6}$ and April P. Carson ${ }^{1, *}$ \\ ${ }^{1}$ University of Alabama at Birmingham, Birmingham, AL, USA \\ ${ }^{2}$ Kaiser Permanente Division of Research, Oakland, CA, USA \\ ${ }^{3}$ Intramural Research Program, National Institute on Aging, Bethesda, MD, USA \\ ${ }^{4}$ Center for Observational Research, Amgen Inc., Thousand Oaks, CA, USA \\ ${ }^{5}$ Global Clinical Development, Cardiovascular and Metabolic Therapeutic Area, Amgen Inc., Thousand Oaks, CA, USA \\ ${ }^{6}$ Icahn School of Medicine at Mount Sinai, New York, NY, USA
}

(Received February 19, 2020; Final Revision December 15, 2020; Accepted January 6, 2021; First Published Online February 10, 2021)

\begin{abstract}
Objectives: It is uncertain if long-term levels of low-density lipoprotein-cholesterol (LDL-C) affect cognition in middle age. We examined the association of LDL-C levels over 25 years with cognitive function in a prospective cohort of black and white US adults. Methods: Lipids were measured at baseline (1985-1986; age: 18-30 years) and at serial examinations conducted over 25 years. Time-averaged cumulative LDL-C was calculated using the area under the curve for 3,328 participants with $\geq 3$ LDL-C measurements and a cognitive function assessment. Cognitive function was assessed at the Year 25 examination with the Digit Symbol Substitution Test [DSST], Rey Auditory Visual Learning Test [RAVLT], and Stroop Test. A brain magnetic resonance imaging (MRI) sub-study $(N=707)$ was also completed at Year 25 to assess abnormal white matter tissue volume (AWMV) and gray matter cerebral blood flow volume (GM-CBFV) as secondary outcomes. Results: There were $15.6 \%, 32.9 \%, 28.9 \%$, and $22.6 \%$ participants with timeaveraged cumulative LDL-C $<100 \mathrm{mg} / \mathrm{dL}, 101-129 \mathrm{mg} / \mathrm{dL}, 130-159 \mathrm{mg} / \mathrm{dL}$, and $\geq 160 \mathrm{mg} / \mathrm{dL}$, respectively.

Standardized differences in all cognitive function test scores ranged from $0.16 S D$ lower to $0.09 S D$ higher across timeaveraged LDL-C categories in comparison to those with LDL-C $<100 \mathrm{mg} / \mathrm{dL}$. After covariate adjustment, participants with higher versus lower time-averaged LDL-C had a lower RAVLT score ( $p$-trend $=0.02$ ) but no differences were present for DSST, Stroop Test, AWMV, or GM-CBFV. Conclusion: Cumulative LDL-C was associated with small differences in memory, as assessed by RAVLT scores, but not other cognitive or brain MRI measures over 25 years of follow-up.
\end{abstract}

Keywords: Cholesterol, LDL, Lipids, Cognition, Cohort studies

\section{INTRODUCTION}

The prevalence of low-density cholesterol (LDL-C) $\geq 3.4$ $\mathrm{mmol} / \mathrm{L}$ declined from $42.9 \%$ in $1999-2000$ to $29.4 \%$ in 2015-2016 among US adults, paralleling observed increases in the use of lipid-lowering medications (Ford \& Capewell, 2013; Virani et al., 2020). In the Cholesterol Treatment Trialists' Collaboration, the risk of a major vascular event was $21 \%$ lower per $1 \mathrm{mmol} / \mathrm{L}$ reduction in LDL-C with statin use (Cholesterol Treatment Trialists et al., 2012). Although

\footnotetext{
*Correspondence and reprint requests to: April P. Carson, $\mathrm{PhD}$, Department of Epidemiology, University of Alabama at Birmingham, 1665 University Boulevard, Birmingham, AL 35233, USA.

E-mail: apcarson@uab.edu

MTM is currently affiliated with Kaiser Permanente Southern California, Pasadena, CA, USA.
}

the cardiovascular benefits of LDL-C lowering are well recognized, there were initial concerns raised regarding the potential for adverse cognitive effects associated with lowering LDL-C with lipid-lowering therapies. Findings from animal models suggested that statins altered the composition of brain lipids (Vecka et al., 2004) and there were early reports of adverse events related to memory and cognition in postmarket surveillance ("FDA Drug Safety Communication: important safety label changes to cholesterol-lowering statin drugs," 2012). With findings from randomized clinical trials showing no difference in cognitive decline for those who received a statin to lower LDL-C compared with those who received a placebo, the current evidence suggests that statin use is not associated with cognition (Heart Protection Study Collaborative, 2002; Newman et al., 2019; Shepherd 
et al., 2002). However, randomized trials provide limited information on the association of long-term LDL-C levels with cognition as they typically provide only a few years of follow-up. Findings from observational studies that have investigated the association of LDL-C with or without lipid-lowering therapy and cognition have been mixed, but these studies have typically relied on a single measure of LDL-C at baseline (Benn, Nordestgaard, Frikke-Schmidt, \& Tybjaerg-Hansen, 2017; Lamar et al., 2019; Mefford et al., 2018; Reitz, Tang, Luchsinger, \& Mayeux, 2004; Shepardson, Shankar, \& Selkoe, 2011; West et al., 2008). It is unclear whether long-term changes or therapeutic reductions in LDL-C over decades may affect cognitive function.

Few studies have examined the association of long-term levels of LDL-C during young adulthood with cognition in middle age. As cognitive decline often develops over an extended period of time (Jack et al., 2010) and LDL-C levels attained may vary over time, we investigated the association of time-averaged LDL-C over 25 years with cognitive function (primary analysis) and with brain tissue and perfusion measures (secondary analysis) in the Coronary Artery Risk Development in Young Adults (CARDIA) study. Given potential associations with LDL-C and cognition, we also examined potential effect modification by statin use ("FDA Drug Safety Communication: important safety label changes to cholesterol-lowering statin drugs," 2012; Stroes, 2005), PCSK9 loss-of-function (LOF) variants (Robinson et al., 2015; Sabatine et al., 2015; Swiger \& Martin, 2015) and Apolipoprotein E (ApoE) phenotypes (Alfred et al., 2014; Bennet et al., 2007; Zhao et al., 2005).

\section{METHODS}

\section{Study Population}

CARDIA is a multicenter prospective study of CVD risk factors among 5,115 adults aged 18-30 years when recruited at baseline in 1985-1986. Participants were recruited at four field centers (Birmingham, AL, Chicago, IL, Minneapolis, $\mathrm{MN}$, and from members of the Kaiser Permanente Medical Care Plan in Oakland, CA). Participants were recruited with equal proportions with respect to age (18-24 years, 25-30 years), race (white, black), sex, and education ( $\leq 12$ years, $>12$ years) at baseline. Follow-up examinations used in the current analysis occurred at 2, 5, 7, 10, 15, 20, and 25 years after baseline. Retention rates have been high (72\% of the surviving cohort attended the Year 25 exam).

For this analysis, we excluded participants who did not attend or died prior to the Year 25 examination $(n=1,617)$, those who attended the Year 25 exam but had $<3$ LDL-C measures $(n=31)$, and those who were missing all cognitive function assessments $(n=139)$ for a final analytic sample of 3,328. Baseline characteristics of participants included and excluded in our analysis are presented in Supplemental Table 1. The primary outcomes were cognitive function measures as assessed by the Digit Symbol Substitution Test (DSST; $N=3,321$ ), the Rey Auditory Verbal Learning Test (RAVLT; $N=3,322)$, and the Stroop Test $(N=3,320)$. A sub-study at the Year 25 examination evaluated brain structural and functional measures using magnetic resonance imaging (MRI). The secondary outcomes from the MRI sub-study included white matter abnormal tissue volume (AWMV; $N=696$ ) and gray matter cerebral blood flow volume (GM-CBFV; $N=539)$.

\section{Data Collection}

Standardized protocols were used for data collection and quality assurance in the CARDIA study (https://www.cardia.dopm.uab. edu/), and all covariates included in the current analysis were collected at the Year 25 examination unless noted otherwise. Education ( $\geq 12$ years, $<12$ years), current smoking (current, former, and never), history of illicit drug use, and alcohol use were self-reported. Alcohol use was categorized as never, moderate (men/women: $>0-14 />0-7$ drinks per week), or heavy (men/women: $>14 />7$ drinks per week). Participation in moderate or vigorous-intensity activities during the prior year was assessed by self-report and calculated as a study-specific measure of exercise units based on the frequency and types of activity using a validated algorithm, with higher scores indicating higher activity levels (Jacobs, Hahn, Haskell, Pirie, \& Sidney, 1989; Parker, Schmitz, Jacobs, Dengel, \& Schreiner, 2007).

The Center for Epidemiological Studies Depression Scale was administered and participants with a score of $\geq 16$ were categorized as having depressive symptoms (Radloff, 1977). Body mass index (BMI) was calculated based on objectively measured height and weight with participants wearing light clothing and no shoes. Systolic blood pressure (SBP) and diastolic blood pressure (DBP) were measured three times using an arm automated blood pressure monitor (Omron HEM907XL) following a $5 \mathrm{~min}$ rest in the seated position, with the last two measurements used to calculate mean SBP and DBP. Statin, non-statin lipid-lowering, antihypertensive, glucose-lowering, and antidepressant medication use was determined by self-report and through a medication inventory during each study examination. Serum creatinine measures were used to calculate the estimated glomerular filtration rate (eGFR) with the Chronic Kidney Disease Epidemiology Collaboration equation with reduced eGFR defined by a level of $<60 \mathrm{ml} / \mathrm{min} / 1.73 \mathrm{~m}^{2}$ (Levey et al., 2009). Diabetes was defined as fasting blood glucose $\geq 126 \mathrm{mg} / \mathrm{dL}, 2$-hr post-challenge glucose from an oral glucose tolerance test $\geq 200$ $\mathrm{mg} / \mathrm{dL}$, hemoglobin A1c $\geq 6.5 \%$, or use of glucose-lowering medications, and includes those with type 1 and type 2 diabetes. History of coronary heart disease (CHD) and history of stroke/transient ischemic attack (TIA) were determined by medical record review and physician adjudication of events prior to the Year 25 exam. ApoE phenotypes (2/2, 3/2, 3/3, $4 / 2,4 / 3$, and 4/4) were measured using blood samples collected at the Year 7 examination using previously defined methods (Kataoka, Paidi, \& Howard, 1994), and categorized as E2, E3, and E4. PCSK9 LOF variants C679X, R46L, and Y142X were genotyped (Fornage \& Doris, 2005) using specimens collected at the Year 20 examination, and carriers were defined as having at least one of these variants. 


\section{Time Averaged LDL-C}

Blood samples were drawn at each CARDIA examination, processed and stored at $-70^{\circ} \mathrm{C}$ until they were analyzed at the Northwest Lipid Research Lab, University of Washington. Total and high-density cholesterol and triglycerides were measured, and LDL-C was calculated at each examination using the Friedewald equation for those with fasting triglycerides $<400 \mathrm{mg} / \mathrm{dL}$. The test-retest correlation was 0.98-0.99 for all lipid measures (Gross et al., 2005). Time-averaged LDL-C levels were estimated for participants with LDL-C measurements at $\geq 3$ CARDIA study visits, and approximately $90 \%$ of participants had 5 or more LDL-C measurements available. Lipid-lowering medication use was not incorporated into the development of time-averaged LDL-C, consistent with prior studies examining other lipids.

Linear mixed models were used to estimate trajectories of LDL-C for each participant, as described previously (Pletcher et al., 2010). Briefly, group mean trajectories were modeled for each race-sex group. It was assumed that trajectories of LDL-C for each participant had a constant slope within each decade (e.g., 20-29, 30-39) and a random intercept. Individual trajectories were then estimated based on grouplevel mean trajectories and conditional expectations from random effects. Next, the area under the curve was calculated for each participant's LDL-C trajectory. Time-averaged LDL-C was then calculated by dividing the area under the curve by a participant's total follow-up time from baseline to their last available LDL-C measurement. Time-averaged LDL-C was categorized as $<100 \mathrm{mg} / \mathrm{dL}, 100-129 \mathrm{mg} / \mathrm{dL}, 130-159$ $\mathrm{mg} / \mathrm{dL}, \geq 160 \mathrm{mg} / \mathrm{dL}$ (Pletcher et al., 2010).

\section{Neurocognitive Function Assessment}

A battery of three standardized tests to measure cognitive function was administered at the Year 25 examination. Repeat measures of cognitive function were not available for this analysis. However, a random review of $5-10 \%$ of cognitive test administration booklets and interview materials was performed for quality assurance. The DSST, a subtest of the Wechsler Adult Intelligence Scale (third edition), assesses an array of cognitive abilities, most prominently visual-motor speed, sustained attention, and working memory (Wechsler, 1997). The range of scores is $0-133$, with higher scores indicating better performance. The RAVLT assesses the ability to memorize and to retrieve words (verbal memory) after several presentations of the word list immediately one after another, and then after a delay of $10 \mathrm{~min}$ (Schmidt, 1996). The range of scores on the delayed test is $0-15$, with higher scores indicating better performance. The Stroop Test includes three subtests that evaluate the ability to view complex visual stimuli and to respond to one stimulus dimension while suppressing the response to the other dimensions (Stroop, 1935). Each subtest is scored by summing the number of errors and the time required for completion. An interference score is calculated by subtracting the score on the incongruent subtest from the congruent subtest. For the Stroop Test, interference scores can be negative or positive and a higher interference score indicates worse performance on the task. For ease of interpretation and comparability across cognitive function measures, all cognitive test scores were transformed into standardized $z$ scores (mean $=0$, standard deviation $=1$ ). For the DSST and RAVLT, positive values indicate better performance and negative values indicate worse performance. For the Stroop Test, positive values indicate worse performance and negative values indicate better performance.

\section{Brain Measures}

Brain MRI was performed in a subsample of participants at Year 25 (Launer et al., 2015) and was used to assess AWMV (Gunning-Dixon \& Raz, 2000) and GM-CBFV (Appelman, van der Graaf, Vincken, Mali, \& Geerlings, 2010; Moser et al., 2012) which have been linked to subtle deficits in cognitive function. Participants enrolled in the sub-study were balanced with respect to race and sex and excluded those with a contraindication to MRI or with a body size too large for the MRI tube bore. Brain MRI was acquired on 3-T MR scanners located proximal to three CARDIA clinic sites (California: Siemens 3T Tim Trio/VB 15 platform; Minnesota: Siemens 3T Tim Trio/VB 15 platform and Birmingham: Philips 3T Achieva/2.6.3.6 platform). The MRI Reading Center, located at the University of Pennsylvania, worked in collaboration with the MRI field centers to train technologists to follow standardized protocols, and transfer MRI data to a central archive located at the Reading Center. To evaluate scanner stability and image distortion prior to site acceptance and quarterly thereafter, each MRI field center followed standard quality assurance protocols developed for the Functional Bioinformatics Research Network, and the Alzheimer's disease (AD) Neuroimaging Initiative. Using scans from three persons measured three times at each field center, the technical error of measurement was $27.8 \%$ for AWMV and $7.3 \%$ for GM-CBFV (Launer et al., 2015). AWMV was analyzed as the proportion above/below the 85th percentile of the observed distribution, with the top $15 \%$ indicative of high AWMV, and the bottom $85 \%$ designated low AWMV, consistent with a previous CARDIA analysis on white matter tissue volume (Zhu et al., 2015). GM-CBFV was analyzed as the proportion above/below the 15th percentile of the observed distribution, with the bottom $15 \%$ indicative of decreased GM-CBFV, and the top $85 \%$ indicative of non-decreased GM-CBFV, also consistent with the previous CARDIA analysis (Zhu et al., 2015).

\section{Statistical Analysis}

Participant characteristics were calculated by the level of timeaveraged LDL-C categories. A statistical test for trend across increasing levels of time-averaged LDL-C was calculated for each covariate by modeling LDL-C category as an ordinal variable and examining the association between LDL-C and each covariate, separately. The mean level of LDL-C and the percentage of participants taking a statin and other lipid-lowering medications, separately, was calculated for each study exam (at 
baseline and 2, 5, 7, 10, 15, 20, and 25 years following baseline) across levels of time-averaged LDL-C. Mean and standardized mean cognitive function scores were calculated for participants by level of time-averaged LDL-C. Using a generalized linear model, we estimated crude and adjusted mean differences in standardized cognitive function scores (DSST, RAVLT, and Stroop Test, separately) for participants with higher time-averaged LDL-C versus the lowest category (LDL-C $<100 \mathrm{mg} / \mathrm{dL}$ ). Model 1 included adjustment for age, race, sex, and education. Model 2 included adjustment for covariates in Model 1 and BMI, diabetes, smoking, alcohol use, physical activity, SBP and DBP, use of antihypertensive medications, reduced eGFR, depressive symptoms, history of illicit drug use, and history of CHD and stroke/TIA. Model 3 included adjustment for covariates in Model 2 and statin, other lipid-lowering medication, and antidepressant medication use. Model covariates were chosen a priori based on previous studies examining the association between LDL-C and cognition, as well as clinical judgment (Gencer et al., 2020; Lamar et al., 2019; Yaffe et al., 2014). Tests for linear trend across LDL-C categories were calculated by modeling the LDL-C category as an ordinal variable.

To test for effect modification, in fully adjusted models we included statin use as a main effect and an interaction term between LDL-C and statin use. We repeated this step to test for effect modification by (1) PCSK9 LOF variants and (2) apoE phenotypes. Finally, we examined associations of time-averaged LDL-C categories with high AWMV and low GM-CBFV, separately, using Poisson regression with robust error variance to obtain prevalence ratios in models with progressive adjustment as described above.

Missing data were minimal for all covariates (Supplemental Table 2), and a complete case analysis was used. For models with interaction terms for PCSK9 variants and apoE phenotypes, a complete case analysis was also used which resulted in more exclusions because more participants were missing data for those variables. In a sensitivity analysis, ApoE phenotype was included as a covariate in fully adjusted models for each cognitive function test. A separate sensitivity analysis calculated LDL-C using the MartinHopkins method which has been shown to have greater concordance with direct measurement than the Friedewald equation (Martin et al., 2013). Analyses were repeated to obtain mean and standardized mean cognitive function scores across levels of time-averaged LDL-C using the Martin-Hopkins method. Two-sided $p$-values of $<0.05$ were considered statistically significant. Analyses were performed using SAS 9.4 (SAS Institute, Cary, NC).

\section{Standard Protocol Approvals, Registrations, and Patient Consents}

The Institutional Review Boards for the protection of human subjects at all study sites provided approval for all aspects of the CARDIA study and written informed consent was obtained from all participants at each study visit.

\section{Data Availability}

CARDIA study datasets may be requested from the NHLBI Biologic Specimen and Data Repository Information Coordinating Center (BioLINCC) at https://biolincc.nhlbi. nih.gov/home/. These are available by application through the BioLINCC website and are subject to established review processes for their distribution to investigators.

\section{RESULTS}

Overall, $15.6 \%, 32.9 \%, 28.9 \%$, and $22.6 \%$ of participants had time-averaged cumulative LDL-C $<100 \mathrm{mg} / \mathrm{dL}, 101-129 \mathrm{mg} /$ $\mathrm{dL}, 130-159 \mathrm{mg} / \mathrm{dL}$, and $\geq 160 \mathrm{mg} / \mathrm{dL}$, respectively (Table 1 ). Participants with higher versus lower time-averaged LDL-C were older, less likely to be female, less likely to be black, and more likely to have $\geq 12$ years of education. Additionally, participants with higher versus lower time-averaged LDL-C were more likely to have a history of illicit drug use, diabetes, CHD, stroke/TIA, higher mean SBP and DBP, and to be taking antihypertensive medication, a statin, and other lipid-lowering medications at the Year 25 exam (all $p$-trend $<0.05$ ). Participants with higher versus lower time-averaged LDL-C were less likely to be carriers of PCSK9 LOF variants and have ApoE2 phenotype but were more likely to have ApoE4 phenotype (all $p$-trends $<0.001$ ). Mean LDL-C and the percentage of participants taking a statin or other lipid-lowering medication at each exam are listed by time-averaged LDL-C category in Supplemental Table 3.

Mean and standardized mean cognitive function scores indicated worse performance among participants with higher versus lower time-averaged LDL-C on the DSST ( $p$-trend $<0.001$ ), RAVLT $(p$-trend $=0.002)$, and the Stroop Test $(p$-trend $=0.01)$ (Table 2, Central Illustration). There were no differences in DSST or Stroop Test scores ( $p$-trend $=0.69$ and 0.30 , respectively) comparing participants with higher versus lower timeaveraged LDL-C after multivariable adjustment (Table 3). Participants with higher time-averaged LDL-C had a lower RAVLT score ( $p$-trend $=0.02$ ). In a sensitivity analyses with additional adjustment for statin use at exam years 15 and 20 and, separately, for ApoE phenotypes, results were similar for the DSST, RAVLT, and Stroop Test (data not shown).

Results were consistent among participants taking and not taking statins, as well as those with and without PCSK9 LOF variants (data not shown; all $p$-interaction $>0.10$ for DSST, RAVLT, and Stroop Test). Additionally, there was no effect modification by ApoE phenotypes for the association of timeaveraged LDL-C and the three cognitive function scores (Supplemental Table 4, $p$-interaction $=0.57,0.09$ and 0.63 for DSST, RAVLT, and Stroop Test, respectively). No differences in cognitive function test scores were present across LDL-C categories for participants with ApoE2 or ApoE3 phenotype. However, for participants with ApoE4 phenotype, participants with higher versus lower time-averaged LDL-C had higher DSST scores $(p$-trend $=0.03$ ).

Characteristics of participants included and not included in the MRI analysis are listed in Supplemental Tables 5 and 6. Compared to those excluded, those included were 
Table 1. Characteristics of participants at the Year 25 Exam by category of time-averaged low-density lipoprotein-cholesterol

\begin{tabular}{|c|c|c|c|c|c|}
\hline & \multicolumn{4}{|c|}{ Time-averaged LDL-C levels over follow-up, mg/dL } & \multirow[b]{2}{*}{$p$-trend } \\
\hline & $<100(n=519)$ & $100-129(n=1,094)$ & $130-159(n=961)$ & $\geq 160(n=754)$ & \\
\hline Age, mean $(S D)$ & $46.9(3.2)$ & $49.2(3.5)$ & $51.1(3.1)$ & $52.6(2.5)$ & $<0.001$ \\
\hline Female, \% & $325(62.6 \%)$ & $659(60.2 \%)$ & $520(54.1 \%)$ & $371(49.2 \%)$ & $<0.001$ \\
\hline Black, \% & $302(58.2 \%)$ & $496(45.3 \%)$ & $390(40.6 \%)$ & $339(45.0 \%)$ & $<0.001$ \\
\hline Education $>12$ years, $\%$ & $377(72.6 \%)$ & $855(78.2 \%)$ & $766(79.7 \%)$ & $595(78.9 \%)$ & 0.01 \\
\hline Current smoking, $\%$ & $114(22.0 \%)$ & $183(16.7 \%)$ & $139(14.5 \%)$ & $107(14.2 \%)$ & $<0.001$ \\
\hline \multicolumn{6}{|l|}{ Alcohol use, $\%$} \\
\hline Heavy & $82(15.8 \%)$ & $152(13.9 \%)$ & $135(14.0 \%)$ & $100(13.3 \%)$ & 0.80 \\
\hline Moderate & $217(41.8 \%)$ & $447(40.9 \%)$ & $397(41.3 \%)$ & $301(39.9 \%)$ & \\
\hline Never & $220(42.4 \%)$ & $492(45.0 \%)$ & $427(44.4 \%)$ & $352(46.7 \%)$ & \\
\hline Total physical activity, mean $(S D)$ in exercise units & $338(283.5)$ & $348.8(270.1)$ & $334.7(280.7)$ & $338.9(271.5)$ & 0.60 \\
\hline \multicolumn{6}{|l|}{ BMI category, $\mathrm{kg} / \mathrm{m}^{2}, \%$} \\
\hline$<25$ & $66(12.7 \%)$ & $121(11.1 \%)$ & $115(12.0 \%)$ & $116(15.4 \%)$ & 0.10 \\
\hline $25-29.9$ & $446(85.9 \%)$ & $965(88.2 \%)$ & $833(86.7 \%)$ & $629(83.4 \%)$ & \\
\hline$\geq 30$ & $7(1.3 \%)$ & $7(0.6 \%)$ & $11(1.1 \%)$ & $9(1.2 \%)$ & \\
\hline Total cholesterol, mean $(S D)$ & $162.1(27.0)$ & $184.3(26.8)$ & $197.7(32.6)$ & $216.9(41.0)$ & $<0.001$ \\
\hline HDL-C, mean $(S D)$ & $62.6(21.5)$ & $58.9(18.0)$ & $56.8(16.5)$ & $54.8(15.9)$ & $<0.001$ \\
\hline Triglycerides, mean $(S D)$ & $94.2(70.7)$ & $110.0(88.5)$ & $115.4(71.8)$ & $132.3(106.4)$ & $<0.001$ \\
\hline CES-D $\geq 16, \%$ & $102(19.7 \%)$ & $188(17.2 \%)$ & $145(15.1 \%)$ & $124(16.4 \%)$ & 0.10 \\
\hline History of illicit drug use, $\%$ & $350(67.4 \%)$ & $753(68.8 \%)$ & $706(73.5 \%)$ & $555(73.6 \%)$ & 0.002 \\
\hline Diabetes, $\%$ & $50(9.6 \%)$ & $124(11.3 \%)$ & $114(11.9 \%)$ & $123(16.3 \%)$ & $<0.001$ \\
\hline History of CHD*, \% & $0(0.0 \%)$ & $9(0.8 \%)$ & $16(1.7 \%)$ & $26(3.4 \%)$ & $<0.001$ \\
\hline History of stroke/TIA*, \% & $2(0.4 \%)$ & $10(0.9 \%)$ & $6(0.6 \%)$ & $14(1.9 \%)$ & 0.02 \\
\hline $\mathrm{eGFR} \leq 60 \mathrm{ml} / \mathrm{min} / 1.73 \mathrm{~m}^{2}, \%$ & $8(1.5 \%)$ & $13(1.2 \%)$ & $19(2.0 \%)$ & $15(2.0 \%)$ & 0.24 \\
\hline $\mathrm{SBP}$, mean $(S D)$ & $117.4(15.8)$ & $118.8(16.4)$ & $120.1(15.8)$ & $121.0(15.8)$ & $<0.001$ \\
\hline $\mathrm{DBP}$, mean $(S D)$ & $73.8(11.4)$ & $74.3(11.8)$ & $75.0(10.7)$ & $75.5(10.6)$ & 0.003 \\
\hline Antihypertensive medication use, $\%$ & $116(22.4 \%)$ & $236(21.6 \%)$ & $277(28.8 \%)$ & $258(34.2 \%)$ & $<0.001$ \\
\hline Statin Use, \% & $13(2.5 \%)$ & $63(5.8 \%)$ & $146(15.2 \%)$ & $219(29.0 \%)$ & $<0.001$ \\
\hline Other lipid-lowering medication, $\%$ & $3(0.6 \%)$ & $16(1.5 \%)$ & $30(3.1 \%)$ & $26(3.4 \%)$ & $<0.001$ \\
\hline Antidepressant medication use, $\%$ & $56(10.8 \%)$ & $124(11.3 \%)$ & $121(12.6 \%)$ & $98(13 \%)$ & 0.14 \\
\hline PCSK9 LOF variant carrier & $32(6.2 \%)$ & $28(2.6 \%)$ & $21(2.2 \%)$ & $5(0.7 \%)$ & $<0.001$ \\
\hline \multicolumn{6}{|l|}{ Apolipoprotein E phenotypes $\$$} \\
\hline E2 & $156(30.1 \%)$ & $156(14.3 \%)$ & $90(9.4 \%)$ & $38(5.0 \%)$ & $<0.001$ \\
\hline E3 & $200(38.5 \%)$ & $554(50.6 \%)$ & $486(50.6 \%)$ & $373(49.5 \%)$ & \\
\hline E4 & $90(17.3 \%)$ & $259(23.7 \%)$ & $269(28.0 \%)$ & $248(32.9 \%)$ & \\
\hline Unknown & $73(14.1 \%)$ & $125(11.4 \%)$ & $116(12.1 \%)$ & $95(12.6 \%)$ & \\
\hline
\end{tabular}

Abbreviations: BMI, body mass index; CES-D, Centers for Epidemiological Studies Depression Scale; CHD, coronary heart disease; DBP, diastolic blood pressure; eGFR, estimated glomerular filtration rate; HDL-C, high-density lipoprotein-cholesterol; LDL-C, low-density lipoprotein-cholesterol; LOF, lossof-function; PCSK9, proprotein convertase subtilisin/kexin type 9; SD, standard deviation; SBP, systolic blood pressure; TIA, transient ischemic attack.

Numbers in table are $n(\%)$ unless noted otherwise.

*History of CHD and History of Stroke/TIA determined by adjudicated events prior to Year 25 examination.

$\dagger$ Obtained at Year 20 follow-up (2005-2006).

¥ Obtained at Year 7 follow-up (1992-1993).

similar in age but had higher proportions of men and white participants across LDL-C categories. Among those included in the analysis, the prevalence of high AWMV was 9.3\%, $17.3 \%, 14.8 \%$, and $13.5 \%$ for those with time-averaged cumulative LDL-C $<100 \mathrm{mg} / \mathrm{dL}, \quad 100-129 \mathrm{mg} / \mathrm{dL}$, $130-159 \mathrm{mg} / \mathrm{dL}$, and $\geq 160 \mathrm{mg} / \mathrm{dL}$, respectively. The prevalence of decreased GM-CBFV was $9.0 \%, 6.7 \%, 14.5 \%$, and $16.2 \%$ for those with time-averaged cumulative LDL-C $<100$ $\mathrm{mg} / \mathrm{dL}, 100-129 \mathrm{mg} / \mathrm{dL}, 130-159 \mathrm{mg} / \mathrm{dL}$, and $\geq 160 \mathrm{mg} / \mathrm{dL}$, respectively. There was no evidence of trends for high AWMV or low GM-CBFV across levels of time-averaged LDL-C after multivariable adjustment (Table 4).
A cross-tabulation of participants categorized into timeaveraged LDL-C categories using both the Friedewald and Martin-Hopkins equations is presented in Supplemental Table 7. Using time-averaged LDL-C calculated using the Martin-Hopkins equation, mean and standardized mean cognitive function scores indicated worse performance among participants with higher versus lower time-averaged LDL-C on the DSST ( $p$-trend $<0.001)$, RAVLT ( $p$-trend $<$ $0.001)$, and the Stroop Test ( $p$-trend $=0.01)$ (Supplemental Table 8). In addition, participants with higher time-averaged LDL-C had a lower RAVLT score after multivariable adjustment $(p$-trend $=0.002)($ Supplemental Table 9$)$. 
Table 2. Mean and standardized mean cognitive function scores by time-averaged LDL-C category using a generalized linear model

\begin{tabular}{|c|c|c|c|c|c|c|}
\hline & \multirow[b]{2}{*}{$N$} & \multicolumn{4}{|c|}{ Average LDL-C levels over follow-up, mg/dL } & \multirow[b]{2}{*}{$p$-trend } \\
\hline & & $<100$ & $100-129$ & $130-159$ & $\geq 160$ & \\
\hline Test & \multicolumn{6}{|c|}{ Mean test score $(S D)^{*}$} \\
\hline DSST (digits correctly substituted, 0-133) & 3321 & $70.6(16.8)$ & $71.1(16.6)$ & $70.4(15.6)$ & $68.0(15.1)$ & $<0.001$ \\
\hline RAVLT (words correctly recalled, $0-15$ ) & 3322 & $9.0(2.0)$ & $9.1(1.9)$ & $9.0(1.9)$ & $8.7(1.9)$ & 0.002 \\
\hline $\begin{array}{l}\text { Stroop Test (seconds to correctly name colors }+ \text { number of } \\
\text { errors, } 1-160 \text { ) }\end{array}$ & 3320 & $22.6(11.8)$ & $22.2(10.5)$ & $22.4(9.8)$ & $24.0(12.1)$ & 0.01 \\
\hline Test & \multicolumn{6}{|c|}{ Standardized mean test score ${ }^{*}(S D)$} \\
\hline DSST & 3321 & $0.03(1.05)$ & $0.06(1.03)$ & $0.02(0.97)$ & $-0.14(0.94)$ & $<0.001$ \\
\hline RAVLT & 3322 & $0.02(1.05)$ & $0.05(1.00)$ & $0.03(0.98)$ & $-0.13(1.00)$ & 0.002 \\
\hline Stroop Test & 3320 & $-0.01(1.08)$ & $-0.04(0.96)$ & $-0.03(0.90)$ & $0.11(1.11)$ & 0.01 \\
\hline
\end{tabular}

Abbreviations: DSST, Digit Symbol Substitution Test; LDL-C, low-density lipoprotein-cholesterol; RAVLT, Rey Auditory Verbal Learning Test; SD, standard deviation.

*Lower mean and standardized mean scores indicate worse performance on the DSST and RAVLT tests, but better performance on the Stroop Test.

Table 3. Mean differences in standardized cognitive function scores* (95\% confidence intervals) associated with time-averaged LDL-C category using a generalized linear model

\begin{tabular}{|c|c|c|c|c|c|c|}
\hline & & \multicolumn{4}{|c|}{ Average LDL-C levels over follow-up, mg/dL } & \multirow[b]{3}{*}{$p$-trend ${ }^{+}$} \\
\hline & & $<100$ & $100-129$ & $130-159$ & $\geq 160$ & \\
\hline & & \multicolumn{4}{|c|}{ Mean difference ( $95 \%$ confidence interval) } & \\
\hline \multirow[t]{3}{*}{ DSST } & Model 1 & Ref & $\begin{array}{c}-0.03 \\
(-0.13,0.07)\end{array}$ & $\begin{array}{l}0.00 \\
(-0.11,0.10)\end{array}$ & $\begin{array}{l}-0.05 \\
(-0.17,0.07)\end{array}$ & 0.55 \\
\hline & Model 2 & Ref & $\begin{array}{c}-0.03 \\
(-0.12,0.07)\end{array}$ & $\begin{array}{l}0.01 \\
(-0.10,0.11)\end{array}$ & $\begin{array}{l}-0.03 \\
(-0.15,0.08)\end{array}$ & 0.79 \\
\hline & Model 3 & Ref & $\begin{array}{c}-0.03 \\
(-0.12,0.07)\end{array}$ & $\begin{array}{l}0.00 \\
(-0.10,0.11)\end{array}$ & $\begin{array}{l}-0.04 \\
(-0.16,0.08)\end{array}$ & 0.69 \\
\hline \multirow[t]{3}{*}{ RAVLT } & Model 1 & Ref & $\begin{array}{c}-0.06 \\
(-0.17,0.04)\end{array}$ & $\begin{array}{l}-0.07 \\
(-0.18,0.04)\end{array}$ & $\begin{array}{l}-0.16 \\
(-0.28,-0.04)\end{array}$ & 0.02 \\
\hline & Model 2 & Ref & $\begin{array}{c}-0.06 \\
(-0.16,0.04)\end{array}$ & $\begin{array}{l}-0.06 \\
(-0.17,0.05)\end{array}$ & $\begin{array}{l}-0.14 \\
(-0.27,-0.02)\end{array}$ & 0.03 \\
\hline & Model 3 & Ref & $\begin{array}{c}-0.06 \\
(-0.16,0.04)\end{array}$ & $\begin{array}{l}-0.07 \\
(-0.18,0.04)\end{array}$ & $\begin{array}{l}-0.16 \\
(-0.28,-0.03)\end{array}$ & 0.02 \\
\hline \multirow[t]{3}{*}{ Stroop Test } & Model 1 & Ref & $\begin{array}{c}0.03 \\
(-0.07,0.14)\end{array}$ & $\begin{array}{l}0.06 \\
(-0.05,0.17)\end{array}$ & $\begin{array}{l}0.09 \\
(-0.03,0.22)\end{array}$ & 0.13 \\
\hline & Model 2 & Ref & $\begin{array}{c}0.03 \\
(-0.08,0.13)\end{array}$ & $\begin{array}{l}0.05 \\
(-0.07,0.16)\end{array}$ & $\begin{array}{l}0.08 \\
(-0.05,0.20)\end{array}$ & 0.22 \\
\hline & Model 3 & Ref & $\begin{array}{c}0.02 \\
(-0.08,0.13)\end{array}$ & $\begin{array}{l}0.04 \\
(-0.07,0.16)\end{array}$ & $\begin{array}{l}0.07 \\
(-0.06,0.19)\end{array}$ & 0.30 \\
\hline
\end{tabular}

Abbreviations: DSST, Digit Symbol Substitution Test; LDL, low-density lipoprotein-cholesterol; RAVLT, Rey Auditory Verbal Learning Test. Model 1 adjusts for age, race, sex, and education.

Model 2 adjusts for Model 1 covariates plus body mass index, diabetes, smoking status, alcohol use, physical activity, systolic and diastolic blood pressure and use of antihypertensive medications, estimated glomerular filtration rate, depressive symptoms, history of illicit drug use, history of coronary heart disease, and history of stroke/transient ischemic attack.

Model 3 adjusts for Model 2 covariates plus statin use, other lipid-lowering medication use, and antidepressant medication use.

*Lower standardized mean scores indicate worse performance on the DSST and RAVLT tests, but better performance on the Stroop Test.

$\dagger$ Calculated using an interaction term between LDL-C category (ordinal) and each variable, separately.

\section{DISCUSSION}

In this retrospective analysis of a prospective cohort of young black and white US adults followed through middle age, mean differences in cognitive function test scores across time-averaged cumulative LDL-C categories were small. In unadjusted analysis, adults with higher versus lower time-averaged LDL-C had worse performance on the DSST, RAVLT, and Stroop Test. However, after multivariable adjustment, higher time-averaged LDL-C was associated with lower RAVLT scores only. For brain tissue and perfusion, time-averaged LDL-C was not associated with high AWMV or decreased GM-CBFV. 
Table 4. Prevalence ratios and $95 \%$ confidence intervals for high abnormal white matter tissue volume and low gray matter cerebral blood flow volume associated with time-averaged LDL-C category

\begin{tabular}{|c|c|c|c|c|c|c|}
\hline & & \multicolumn{4}{|c|}{ Average LDL-C levels over follow-up, mg/dL } & \multirow[b]{4}{*}{$p$-trend ${ }^{\dagger}$} \\
\hline & & $<100$ & $100-129$ & $130-159$ & $\geq 160$ & \\
\hline & & $(n=107)$ & $(n=225)$ & $(n=209)$ & $(n=155)$ & \\
\hline & & \multicolumn{4}{|c|}{ Prevalence ratio (95\% confidence interval) } & \\
\hline \multirow[t]{6}{*}{ High AWMV } & Model 1 & Ref & $1.67(0.85-3.27)$ & $1.38(0.68-2.79)$ & $1.17(0.54-2.53)$ & 0.69 \\
\hline & Model 2 & Ref & $1.50(0.76-2.96)$ & $1.28(0.62-2.62)$ & $1.16(0.52-2.61)$ & 0.86 \\
\hline & Model 3 & Ref & $1.54(0.77-3.05)$ & $1.27(0.62-2.62)$ & $1.12(0.47-2.63)$ & 0.77 \\
\hline & & $<100$ & $100-129$ & $130-159$ & $\geq 160$ & \\
\hline & & $(n=78)$ & $(n=179)$ & $(n=152)$ & $(n=130)$ & \\
\hline & & \multicolumn{4}{|c|}{ Prevalence ratio ( $95 \%$ confidence interval) } & \\
\hline \multirow[t]{3}{*}{ Low GM-CBFV } & Model 1 & Ref & $0.64(0.26-1.56)$ & $1.13(0.50-2.55)$ & $1.11(0.48-2.60)$ & 0.25 \\
\hline & Model 2 & Ref & $0.57(0.23-1.45)$ & $1.01(0.41-2.48)$ & $0.84(0.33-2.13)$ & 0.66 \\
\hline & Model 3 & Ref & $0.60(0.24-1.49)$ & $1.08(0.44-2.61)$ & $1.00(0.39-2.51)$ & 0.35 \\
\hline
\end{tabular}

Abbreviations: GM-CBFV, gray matter cerebral blood flow volume; LDL-C, low-density lipoprotein-cholesterol; MRI, magnetic resonance imaging; AWMV, abnormal white matter volume.

Model 1 adjusts for age, race, sex, and education.

Model 2 adjusts for Model 1 covariates plus body mass index, diabetes, smoking status, alcohol use, physical activity, systolic and diastolic blood pressure and use of antihypertensive medications, estimated glomerular filtration rate, depressive symptoms, history of illicit drug use, history of coronary heart disease, and history of stroke/transient ischemic attack.

Model 3 adjusts for Model 2 covariates plus statin use, other lipid-lowering medication use, and antidepressant medication use.

In the current study, after adjustment for potential confounders, standardized differences in cognitive function test scores ranged from $0.16 S D$ lower to $0.09 S D$ higher across time-averaged LDL-C categories in comparison to those with LDL-C $<100 \mathrm{mg} / \mathrm{dL}$. Higher time-averaged LDL-C was associated with lower RAVLT scores. The RAVLT focuses on the ability to memorize and retrieve words (verbal memory), including delayed recall. Previous evidence from biological studies of lipid dysregulation in AD suggests memory impairment is a strong indicator and early sign of AD (Bjorkhem, Cedazo-Minguez, Leoni, \& Meaney, 2009; Kunkle et al., 2019; Reitz, 2013). Additionally, findings from the Northern Manhattan Study showed that higher LDL-C increased the risk for incident vascular dementia (Reitz et al., 2004). In a study of HIV-infected men taking antiretroviral therapy, higher levels of LDL-C were associated with greater cognitive decline assessed by a cognitive summary score including the RAVLT (Mukerji et al., 2016). In contrast, a positive association between LDL-C levels and cognitive measures of learning and verbal fluency was reported in a cross-sectional analysis of the Hispanic Community Health Study/Study of Latinos (2008-2011) (Lamar et al., 2019). The overall evidence is conflicting about the relationship between serum lipids and brain lipids, and how these two are interconnected with cognition. Despite dietary cholesterol not crossing the blood-brain barrier, increases in cholesterol can lead to the formation of cholesterol metabolites, proinflammatory mediators, and antioxidant processes, that signal the brain and impact cognition (Schreurs, 2010). Although it is difficult to posit a definitive mechanism of action, research in animals and humans has shown that cholesterol levels are related to memory and disturbances to cholesterol can result in a range of effects on cognition (Schreurs, 2010). Differences between the current study and previous observational studies include the frequency and timing of LDL-C measures, the age of the participants included, cognitive function tests utilized, and cognitive abilities examined, which may partially explain the variability in findings. An important next step is to delineate specific aspects of memory that may be influenced by long-term lipid levels versus other markers of health and aging. Additionally, future studies should investigate whether LDL particle size and LDL-C/ LDL particle concentration discordance may contribute to mixed findings for lipids with cognition.

Past randomized trials have investigated the effects of pharmacologic therapies that lower LDL-C (i.e., statins and PCSK9 inhibitors) on cognition (Giugliano et al., 2017; Robinson et al., 2017; Shepherd et al., 2002). Findings in the current study are consistent with randomized trial data, where there was no evidence of differences in cognitive impairment across high or low levels of LDL-C among participants taking and not taking statins. In addition, in the current study, there was no association between LDL-C levels and cognition among participants with or without PCSK9 LOF variants. This is consistent with a retrospective analysis of the REasons for Geographic And Racial Differences in Stroke (REGARDS) study, where PCSK9 LOF variants and resulting low levels of LDL-C, were not associated with impairment measured by a battery of validated cognitive assessments (Mefford et al., 2018). Among participants in 
the current study with apoE4 phenotype, those with higher versus lower LDL-C had a better DSST score, however these differences were small. Given the evidence that apoE4 influences LDL-C levels (Bennet et al., 2007), cognition in middle age (Zhao et al., 2005), and may result in greater cognitive decline later in life (Schiepers et al., 2012), this may require further investigation.

High levels of LDL-C that are still within the range considered normal can result in endothelial dysfunction (Steinberg et al., 1997) and brain abnormalities including compromised white matter structural integrity (Williams et al., 2013). In the current study, time-averaged LDL-C was not associated with high AWMV or decreased GM-CBFV. Previous research examining the association of LDL-C levels with brain structure and perfusion has been limited, with reports of both no association and an association between lower LDL-C and worse white matter microstructural integrity (Debette et al., 2011; Power et al., 2017). In the Memory in Diabetes extension of the Action to Control Cardiovascular Risk in Diabetes randomized trial $(n=236)$, intensive LDL-C lowering versus placebo was not associated with total brain volume (Williamson et al., 2014). Additionally, a study of neural correlates and modifiers of cognitive aging reported LDL-C was not associated with white matter hyperintensity among adults aged 44-77 years (Raz, Yang, Dahle, \& Land, 2012). White matter hyperintensities in the brain and lower levels of cerebral blood flow have been associated with lower cognitive function (Appelman et al., 2010; Gunning-Dixon \& Raz, 2000; Moser et al., 2012), and may be early indicators of decline in cognitive performance.

Strengths of the current study include using a contemporary prospective cohort of middle-aged adults with 25 years of follow-up. Serial measurements of lipid levels at up to eight study examinations allowed us to examine the cumulative exposure of LDL-C across the life course. There are potential limitations to note as well. Cognitive function scores were assessed crosssectionally at Year 25 and we were unable to assess longitudinal changes in cognition from baseline. As the CARDIA study enrolled young adults at baseline, the population at 25 years post-baseline may still be too young to detect measurable differences in cognition. Although the majority of participants from the Year 25 exam were included in the primary analysis, there were differences with respect to race, education, and current smoking status for those included and excluded, which may have impacted our overall results. Additionally, measures of AWMV and GM-CBFV were only available among CARDIA participants in a sub-study at the Year 25 examination, and these measurements were not available at baseline. The time-averaged LDL-C measure reflected long-term LDL levels only, and not LDL-C variability specifically. Therefore, this may have limited our ability to detect associations between time-averaged categories, although variability in LDL-C levels was present within all categories and not specific to higher or lower time-averaged LDL-C. While we included several covariates of interest in the fully adjusted models, approximately $30 \%$ of the variability in the associations between LDL-C and cognitive function was explained by these variables and residual and unmeasured confounding remain a possibility. Finally, PCSK9 genotyping and ApoE phenotypes were only measured in subsamples of CARDIA participants, and this may have limited the statistical power to detect differences across categories of time-averaged LDL-C. Given the smaller sample size available to test effect modification by PCSK9 variants and ApoE phenotypes, we interpret these results cautiously.

In conclusion, mean differences in cognitive function test scores across time-averaged LDL-C categories were small among middle-aged black and white US adults. We provide evidence that higher long-term levels of LDL-C were associated with slightly worse memory performance but not other cognitive measures over 25 years of follow-up from young adulthood to middle age.

\section{SUPPLEMENTARY MATERIAL}

To view supplementary material for this article, please visit https://doi.org/10.1017/S1355617721000059

\section{ACKNOWLEDGMENTS}

The authors would also like to thank Feng Lin from the University of California at San Francisco for the development of the code for time-averaged cholesterol.

\section{CONFLICT OF INTEREST}

$\mathrm{MM}$ and LC report no disclosures. CEL receives research support from grant HHSN26820130026C. PM receives research support from Amgen, Inc. SS and LJL report no disclosures. KLM, AR, and HK are employed by Amgen, Inc. RSR receives research grant support from Akcea, Amgen, Medicines Company, and Sanofi; he receives honoraria from Akcea, Kowa, and Pfizer; he has ownership in MediMergent; he receives consultant fees from Akcea, Amgen, Regeneron, and Sanofi; he receives other support from UpToDate. APC receives research support from Amgen, Inc.

\section{FUNDING}

The Coronary Artery Risk Development in Young Adults Study (CARDIA) is conducted and supported by the National Heart, Lung, and Blood Institute (NHLBI) in collaboration with the University of Alabama at Birmingham (HHSN268201800005I \& HHSN268201800007I), Northwestern University (HHSN 268201800003I), University of Minnesota (HHSN268 201800006I), and Kaiser Foundation Research Institute (HHSN268201800004I). This manuscript has been reviewed by CARDIA for the scientific content. CARDIA was also partially supported by the Intramural Research Program of the National Institute on Aging (NIA) and an intra-agency agreement between NIA and NHLBI (AG0005). The current secondary data analysis was funded by an academic/industry collaboration between the University of Alabama at Birmingham, Mount Sinai School of Medicine, and Amgen, Inc. 


\section{REFERENCES}

Alfred, T., Ben-Shlomo, Y., Cooper, R., Hardy, R., Cooper, C., Deary, I.J., ... HALCyon Study Team. (2014). Associations between APOE and low-density lipoprotein cholesterol genotypes and cognitive and physical capability: the HALCyon programme. Age (Dordr), 36(4), 9673. doi:10.1007/s11357-014-9673-9

Appelman, A.P., van der Graaf, Y., Vincken, K.L., Mali, W.P., \& Geerlings, M.I. (2010). Combined effect of cerebral hypoperfusion and white matter lesions on executive functioning - The SMART-MR study. Dementia and Geriatric Cognitive Disorders, 29(3), 240-247. doi:10.1159/000289813

Benn, M., Nordestgaard, B.G., Frikke-Schmidt, R., \& TybjaergHansen, A. (2017). Low LDL cholesterol, PCSK9 and HMGCR genetic variation, and risk of Alzheimer's disease and Parkinson's disease: Mendelian randomisation study. BMJ, 357, j1648. doi:10.1136/bmj.j1648

Bennet, A.M., Di Angelantonio, E., Ye, Z., Wensley, F., Dahlin, A., Ahlbom, A., ... Danesh, J. (2007). Association of apolipoprotein E genotypes with lipid levels and coronary risk. JAMA, 298(11), 1300-1311. doi:10.1001/jama.298.11.1300

Bjorkhem, I., Cedazo-Minguez, A., Leoni, V., \& Meaney, S. (2009). Oxysterols and neurodegenerative diseases. Molecular Aspects of Medicine, 30(3), 171-179. doi:10.1016/j.mam.2009.02.001

Cholesterol Treatment Trialists, C., Mihaylova, B., Emberson, J., Blackwell, L., Keech, A., Simes, J., ... Baigent, C. (2012). The effects of lowering LDL cholesterol with statin therapy in people at low risk of vascular disease: meta-analysis of individual data from 27 randomised trials. Lancet, 380(9841), 581-590. doi:10.1016/S0140-6736(12)60367-5

Debette, S., Seshadri, S., Beiser, A., Au, R., Himali, J.J., Palumbo, C., ... DeCarli, C. (2011). Midlife vascular risk factor exposure accelerates structural brain aging and cognitive decline. Neurology, 77(5), 461-468. doi:10.1212/WNL.0b013e318227b227

FDA Drug Safety Communication: important safety label changes to cholesterol-lowering statin drugs ([article online]). (2012). http:// www.fda.gov/Drugs/DrugSafety/ucm293101.htm

Ford, E.S. \& Capewell, S. (2013). Trends in total and low-density lipoprotein cholesterol among U.S. adults: contributions of changes in dietary fat intake and use of cholesterol-lowering medications. PLoS One, 8(5), e65228. doi:10.1371/journal.pone.0065228

Fornage, M. \& Doris, P.A. (2005). Single-nucleotide polymorphism genotyping for disease association studies. Methods in Molecular Medicine, 108, 159-172.

Gencer, B., Mach, F., Guo, J., Im, K., Ruzza, A., Wang, H., ... Investigators, F. (2020). Cognition after lowering LDL-cholesterol with evolocumab. Journal of the American College of Cardiology, 75(18), 2283-2293. doi:10.1016/j.jacc.2020.03.039

Giugliano, R.P., Mach, F., Zavitz, K., Kurtz, C., Im, K., Kanevsky, E., ... Ebbinghaus Investigators. (2017). Cognitive function in a randomized trial of evolocumab. The New England Journal of Medicine, 377(7), 633-643. doi:10.1056/NEJMoa1701131

Gross, M., Steffes, M., Jacobs, D.R., Jr., Yu, X., Lewis, L., Lewis, C.E., \& Loria, C.M. (2005). Plasma F2-isoprostanes and coronary artery calcification: the CARDIA Study. Clinical Chemistry, 51(1), 125-131. doi:10.1373/clinchem.2004.037630

Gunning-Dixon, F.M. \& Raz, N. (2000). The cognitive correlates of white matter abnormalities in normal aging: a quantitative review. Neuropsychology, 14(2), 224-232.

Heart Protection Study Collaborative Group. (2002). MRC/BHF Heart Protection Study of cholesterol lowering with simvastatin in 20,536 high-risk individuals: a randomised placebo-controlled trial. Lancet, 360(9326), 7-22. doi:10.1016/S0140-6736(02) 09327-3

Jack, C.R., Jr., Knopman, D.S., Jagust, W.J., Shaw, L.M., Aisen, P.S., Weiner, M.W., ... Trojanowski, J.Q. (2010). Hypothetical model of dynamic biomarkers of the Alzheimer's pathological cascade. Lancet Neurology, 9(1), 119-128. doi:10. 1016/S1474-4422(09)70299-6

Jacobs, D.R., Jr., Hahn, L.P., Haskell, W.L., Pirie, P., \& Sidney, S. (1989). Validity and reliability of short physical activity history: cardia and the Minnesota heart health program. Journal of Cardiopulmonary Rehabilitation and Prevention, 9(11), 448-459.

Kataoka, S., Paidi, M., \& Howard, B.V. (1994). Simplified isoelectric focusing/immunoblotting determination of apoprotein E phenotype. Clinical Chemistry, 40(1), 11-13.

Kunkle, B.W., Grenier-Boley, B., Sims, R., Bis, J.C., Damotte, V., Naj, A.C., ... Pericak-Vance, M.A. (2019). Genetic meta-analysis of diagnosed Alzheimer's disease identifies new risk loci and implicates Abeta, tau, immunity and lipid processing. Nature Genetics, 51(3), 414-430. doi:10.1038/s41588-019-0358-2

Lamar, M., Durazo-Arvizu, R.A., Rodriguez, C.J., Kaplan, R.C., Perera, M.J., Cai, J., ... Daviglus, M.L. (2019). Associations of lipid levels and cognition: findings from the hispanic community health study/study of Latinos. Journal of the International Neuropsychological Society, 1-12. doi:10.1017/ S1355617719001000

Launer, L.J., Lewis, C.E., Schreiner, P.J., Sidney, S., Battapady, H., Jacobs, D.R., . . B Bryan, R.N. (2015). Vascular factors and multiple measures of early brain health: CARDIA brain MRI study. PLoS One, 10(3), e0122138. doi:10.1371/journal.pone.0122138

Levey, A.S., Stevens, L.A., Schmid, C.H., Zhang, Y.L., Castro, A.F., 3rd, Feldman, H.I., ... CKD-EPI (Chronic Kidney Disease Epidemiology Collaboration). (2009). A new equation to estimate glomerular filtration rate. Annals of Internal Medicine, 150(9), 604-612.

Martin, S.S., Blaha, M.J., Elshazly, M.B., Toth, P.P., Kwiterovich, P.O., Blumenthal, R.S., \& Jones, S.R. (2013). Comparison of a novel method vs the Friedewald equation for estimating low-density lipoprotein cholesterol levels from the standard lipid profile. JAMA, 310(19), 2061-2068. doi:10.1001/jama.2013.280532

Mefford, M.T., Rosenson, R.S., Cushman, M., Farkouh, M.E., McClure, L.A., Wadley, V.G., ... Levitan, E.B. (2018). PCSK9 variants, low-density lipoprotein cholesterol, and neurocognitive impairment: reasons for geographic and racial differences in stroke study (REGARDS). Circulation, 137(12), 1260-1269. doi:10.1161/CIRCULATIONAHA.117.029785

Moser, D.J., Boles Ponto, L.L., Miller, I.N., Schultz, S.K., Menda, Y., Arndt, S., \& Nopoulos, P.C. (2012). Cerebral blood flow and neuropsychological functioning in elderly vascular disease patients. Journal of Clinical and Experimental Neuropsychology, 34(2), 220-225. doi:10.1080/13803395.2011.630653

Mukerji, S.S., Locascio, J.J., Misra, V., Lorenz, D.R., Holman, A., Dutta, A., ... Gabuzda, D. (2016). Lipid profiles and APOE4 allele impact midlife cognitive decline in HIV-infected men on antiretroviral therapy. Clinical Infectious Diseases, 63(8), 1130-1139. doi:10.1093/cid/ciw495

Newman, C.B., Preiss, D., Tobert, J.A., Jacobson, T.A., Page, R.L., 2nd, Goldstein, L.B., ... Stroke, C. (2019). Statin safety and associated adverse events: a scientific statement from the American Heart Association. Arteriosclerosis, Thrombosis, and Vascular Biology, 39(2), e38-e81. doi:10.1161/ATV. 0000000000000073 
Parker, E.D., Schmitz, K.H., Jacobs, D.R., Jr., Dengel, D.R., \& Schreiner, P.J. (2007). Physical activity in young adults and incident hypertension over 15 years of follow-up: the CARDIA study. American Journal of Public Health, 97(4), 703-709. doi:10.2105/ AJPH.2004.055889

Pletcher, M.J., Bibbins-Domingo, K., Liu, K., Sidney, S., Lin, F., Vittinghoff, E., \& Hulley, S.B. (2010). Nonoptimal lipids commonly present in young adults and coronary calcium later in life: the CARDIA (Coronary Artery Risk Development in Young Adults) study. Annals of Internal Medicine, 153(3), 137-146. doi:10.7326/0003-4819-153-3-201008030-00004

Power, M.C., Tingle, J.V., Reid, R.I., Huang, J., Sharrett, A.R., Coresh, J., . . Mosley, T.H. (2017). Midlife and late-life vascular risk factors and white matter microstructural integrity: the atherosclerosis risk in communities neurocognitive study. Journal of the American Heart Association, 6(5). doi:10.1161/JAHA.117.005608

Radloff, L.S. (1977). The CES-D scale a self-report depression scale for research in the general population. Applied Psychological Measurement, 1, 385-401.

Raz, N., Yang, Y., Dahle, C.L., \& Land, S. (2012). Volume of white matter hyperintensities in healthy adults: contribution of age, vascular risk factors, and inflammation-related genetic variants. Biochimica et Biophysica Acta, 1822(3), 361-369. doi:10. 1016/j.bbadis.2011.08.007

Reitz, C. (2013). Dyslipidemia and the risk of Alzheimer's disease. Current Atherosclerosis Reports, 15(3), 307. doi:10.1007/ s11883-012-0307-3

Reitz, C., Tang, M.X., Luchsinger, J., \& Mayeux, R. (2004). Relation of plasma lipids to Alzheimer's disease and vascular dementia. Archives of Neurology, 61(5), 705-714. doi:10.1001/ archneur.61.5.705

Robinson, J.G., Farnier, M., Krempf, M., Bergeron, J., Luc, G., Averna, M., ... Odyssey Long Term Investigators. (2015). Efficacy and safety of alirocumab in reducing lipids and cardiovascular events. The New England Journal of Medicine, 372(16), 1489-1499. doi:10.1056/NEJMoa1501031

Robinson, J.G., Rosenson, R.S., Farnier, M., Chaudhari, U., Sasiela, W.J., Merlet, L., . . Kastelein, J.J. (2017). Safety of very low low-density lipoprotein cholesterol levels with alirocumab: pooled data from randomized trials. Journal of the American College of Cardiology, 69(5), 471-482. doi:10.1016/j.jacc.2016.11.037

Sabatine, M.S., Giugliano, R.P., Wiviott, S.D., Raal, F.J., Blom, D.J., Robinson, J., ... Open-Label Study of Long-Term Evaluation against LDL Cholesterol Investigators. (2015). Efficacy and safety of evolocumab in reducing lipids and cardiovascular events. The New England Journal of Medicine, 372(16), 1500-1509. doi:10.1056/NEJMoa1500858

Schiepers, O.J., Harris, S.E., Gow, A.J., Pattie, A., Brett, C.E., Starr, J.M., \& Deary, I.J. (2012). APOE E4 status predicts agerelated cognitive decline in the ninth decade: longitudinal follow-up of the Lothian Birth Cohort 1921. Molecular Psychiatry, 17(3), 315-324. doi:10.1038/mp.2010.137

Schmidt, M. (1996). Rey Auditory Verbal Learning Test: A Handbook. Los Angeles: Western Psychological Services.

Schreurs, B.G. (2010). The effects of cholesterol on learning and memory. Neuroscience \& Biobehavioral Reviews, 34(8), 13661379. doi:10.1016/j.neubiorev.2010.04.010

Shepardson, N.E., Shankar, G.M., \& Selkoe, D.J. (2011). Cholesterol level and statin use in Alzheimer disease: II. Review of human trials and recommendations. Archives of Neurology, 68(11), 1385-1392. doi:10.1001/archneurol. 2011.242
Shepherd, J., Blauw, G.J., Murphy, M.B., Bollen, E.L., Buckley, B.M., Cobbe, S.M., ... Prosper Study Group. Prospective Study of Pravastatin in the Elderly at Risk. (2002). Pravastatin in elderly individuals at risk of vascular disease (PROSPER): a randomised controlled trial. Lancet, 360(9346), 1623-1630. doi:10.1016/ s0140-6736(02)11600-x

Steinberg, H.O., Bayazeed, B., Hook, G., Johnson, A., Cronin, J., \& Baron, A.D. (1997). Endothelial dysfunction is associated with cholesterol levels in the high normal range in humans. Circulation, 96(10), 3287-3293.

Stroes, E. (2005). Statins and LDL-cholesterol lowering: an overview. Current Medical Research and Opinion, 21(Suppl 6), S9-S16. doi:10.1185/030079905X59102

Stroop, J. (1935). Studies of interference in serial verbal reaction. Journal of Experimental Psychology, 18, 643.

Swiger, K.J. \& Martin, S.S. (2015). PCSK9 inhibitors and neurocognitive adverse events: exploring the FDA directive and a proposal for N-of-1 trials. Drug Safety, 38(6), 519-526. doi:10.1007/ s40264-015-0296-6

Vecka, M., Tvrzicka, E., Stankova, B., Novak, F., Novakova, O., \& Zak, A. (2004). Hypolipidemic drugs can change the composition of rat brain lipids. The Tohoku Journal of Experimental Medicine, 204(4), 299-308. doi:10.1620/tjem.204.299

Virani, S.S., Alonso, A., Benjamin, E.J., Bittencourt, M.S., Callaway, C.W., Carson, A.P., ... Stroke Statistics, S. (2020). Heart disease and stroke statistics-2020 update: a report from the American heart association. Circulation, 141(9), e139e596. doi:10.1161/CIR.0000000000000757

Wechsler, D. (1997). Wechsler Adult Intelligence Scale-III (WAISIII). New York: Psychological Corporation.

West, R., Beeri, M.S., Schmeidler, J., Hannigan, C.M., Angelo, G., Grossman, H.T., . . . Silverman, J.M. (2008). Better memory functioning associated with higher total and low-density lipoprotein cholesterol levels in very elderly subjects without the apolipoprotein e4 allele. The American Journal of Geriatric Psychiatry, 16(9), 781-785. doi:10.1097/JGP.0b013e3181812790

Williams, V.J., Leritz, E.C., Shepel, J., McGlinchey, R.E., Milberg, W.P., Rudolph, J.L., ... Salat, D.H. (2013). Interindividual variation in serum cholesterol is associated with regional white matter tissue integrity in older adults. Human Brain Mapping, 34(8), 1826-1841. doi:10. 1002/hbm.22030

Williamson, J.D., Launer, L.J., Bryan, R.N., Coker, L.H., Lazar, R.M., Gerstein, H.C., ... Action to Control Cardiovascular Risk in Diabetes Memory in Diabetes Investigators. (2014). Cognitive function and brain structure in persons with type 2 diabetes mellitus after intensive lowering of blood pressure and lipid levels: a randomized clinical trial. JAMA Internal Medicine, 174(3), 324-333. doi:10. 1001/jamainternmed.2013.13656

Yaffe, K., Vittinghoff, E., Pletcher, M.J., Hoang, T.D., Launer, L.J., Whitmer, R., ... Sidney, S. (2014). Early adult to midlife cardiovascular risk factors and cognitive function. Circulation, 129(15), 1560-1567. doi:10.1161/CIRCULATIONAHA.113.004798

Zhao, J.H., Brunner, E.J., Kumari, M., Singh-Manoux, A., Hawe, E., Talmud, P.J., ... Humphries, S.E. (2005). APOE polymorphism, socioeconomic status and cognitive function in mid-life-the Whitehall II longitudinal study. Social Psychiatry and Psychiatric Epidemiology, 40(7), 557-563. doi:10.1007/s00127-005-0925-y

Zhu, N., Jacobs, D.R., Jr., Schreiner, P.J., Launer, L.J., Whitmer, R.A., Sidney, S., ... Bryan, R.N. (2015). Cardiorespiratory fitness and brain volume and white matter integrity: The CARDIA Study. Neurology, 84(23), 2347-2353. doi:10.1212/WNL. 0000000000001658 